\title{
Neoteric formulas of the monic orthogonal Chebyshev polynomials of the sixth-kind involving moments and linearization formulas
}

\author{
Waleed M. Abd-Elhameed ${ }^{1}$ (I) and Youssri H. Youssri ${ }^{1 *}$ (D)
}

"Correspondence:

youssri@sci.cu.edu.eg

${ }^{1}$ Department of Mathematics,

Faculty of Science, Cairo University,

Giza 12613, Egypt

\section{Springer}

\begin{abstract}
The principal aim of the current article is to establish new formulas of Chebyshev polynomials of the sixth-kind. Two different approaches are followed to derive new connection formulas between these polynomials and some other orthogonal polynomials. The connection coefficients are expressed in terms of terminating hypergeometric functions of certain arguments; however, they can be reduced in some cases. New moment formulas of the sixth-kind Chebyshev polynomials are also established, and in virtue of such formulas, linearization formulas of these polynomials are developed.
\end{abstract}

MSC: Primary 33-XX; secondary 33CXX; 42C05

Keywords: Chebyshev polynomials; Connection coefficients; Zeilberger's and Petkovsek's algorithms; Generalized hypergeometric functions; Moment formulas; Linearization problems

\section{Introduction}

Special functions in general and orthogonal polynomials, in particular, have been used for centuries. These functions and polynomials play essential parts in various disciplines such as theoretical physics, chemistry, and numerical analysis (see, for example, [1-3]). In particular, they occupy significant parts in the solution of differential equations of different types.

It is well known that there are several kinds of Chebyshev polynomials. The one most commonly used is of the first kind, but it is shown that all kinds have their roles to play (see [2]). The four kinds $T_{n}(x), U_{n}(x), V_{n}(x)$ and $W_{n}(x)$ denote, respectively, the first-, second-, third-, and fourth-kind Chebyshev polynomials. They are particular classes of Jacobi polynomials. The first- and second-kind polynomials are ultraspherical polynomials, however, the third- and fourth-kinds are not ultraspherical polynomials. They are special nonsymmetric Jacobi polynomials. The third- and fourth-kind polynomials were first defined by Gautschi (see [4]). He named them Chebyshev polynomials since they have trigonomet-

(c) The Author(s) 2021. This article is licensed under a Creative Commons Attribution 4.0 International License, which permits use, sharing, adaptation, distribution and reproduction in any medium or format, as long as you give appropriate credit to the original author(s) and the source, provide a link to the Creative Commons licence, and indicate if changes were made. The images or other third party material in this article are included in the article's Creative Commons licence, unless indicated otherwise in a credit line to the material. If material is not included in the article's Creative Commons licence and your intended use is not permitted by statutory regulation or exceeds the permitted use, you will need to obtain permission directly from the copyright holder. To view a copy of this licence, visit http://creativecommons.org/licenses/by/4.0/. 
ric definitions, like the first- and second-kinds. There were two other kinds of Chebyshev polynomials introduced by Jamei [5]. He named them Chebyshev polynomials of the fifthand sixth-kinds. Furthermore, he classified them as two half-trigonometric orthogonal polynomials because he could express only the even-order polynomials in trigonometric definitions. Recently, Abd-Elhameed and Youssri found full trigonometric representations for the fifth- and sixth-kinds of any order (see [6, 7]). It should be pointed out here that all the six kinds of Chebyshev polynomials were employed in several applications in differential equations. For some articles concerned with the first four kinds see [8-10], and for some other contributions concerning the fifth- and sixth-kinds see $[6,7,11]$.

Of the important problems related to the topic of special functions are the linearization problems and their related and generalizing problems. In this regard, we mention some of these problems accompanied by some contributions regarding them.

- Linearization problems of different orthogonal polynomials. For example, and in this regard, recently, the author in [12] developed new linearization formulas of generalized Laguerre polynomials. More recently, the authors in [13] established some specific and general linearization formulas of certain classes of Jacobi polynomials. The author in [14] established linearization formulas of certain non-symmetric classes of Jacobi polynomials. The authors in [15] derived some formulas of the linearization coefficients of some classes of Jacobi polynomials.

- Summing finite products of different orthogonal polynomials. This important problem was investigated by some authors. In this regard, the authors in [16] represented certain sums of finite products of Chebyshev polynomials in terms of Chebyshev polynomials. The authors in [17] represented sums of finite products of Chebyshev polynomials of the first-kind and Lucas polynomials in terms of some orthogonal polynomials. Other expressions of sums of finite products of Legendre and Laguerre polynomials were developed in [18]. Recently, the authors in [19] developed new sums of finite products of the second-, third-, and fourth-kind Chebyshev polynomials.

- Connection problems between different orthogonal polynomials. Some studies were devoted to solving these problems via different approaches. For some articles interested in investigating these problems, one can be referred for example to [20, 21].

In this paper, we concentrate on developing some important formulas of Chebyshev polynomials of the sixth-kind including connection and linearization formulas. Regarding the general linearization problem, if we have three polynomial sets $\left\{\phi_{m}(x)\right\}_{m \geq 0},\left\{\psi_{n}(x)\right\}_{n \geq 0}$, and $\left\{\xi_{p}(x)\right\}_{p \geq 0}$, then the problem

$$
\phi_{m}(x) \psi_{n}(x)=\sum_{r=0}^{m+n} L_{r, m, n} \xi_{r}(x)
$$

is called the general linearization problem, and the coefficients $L_{r, m, n}$ are called the linearization coefficients. To solve the linearization problem (1), we should find the coefficients $L_{r, m, n}$. In particular, we have two important problems considered as special cases of problem (1): 
(i) The linearization problem,

$$
\phi_{m}(x) \phi_{n}(x)=\sum_{r=0}^{m+n} M_{r, m, n} \phi_{r}(x)
$$

where $M_{r, m, n}$ are the linearization coefficients to be determined.

(ii) The connection problem,

$$
\phi_{m}(x)=\sum_{r=0}^{m+n} S_{r, m, n} \xi_{r}(x)
$$

where $S_{r, m, n}$ are the connection coefficients to be determined.

Several algorithms were described to solve the connection and linearization problems. In most cases, the connection and linearization coefficients are expressed in terms of hypergeometric functions of certain arguments; see, for example [20, 22-24].

There are extensive studies regarding the first four kinds of Chebyshev polynomials form theoretical and numerical points of view, however, the studies regarding the fifth- and sixth-kinds of Chebyshev polynomials are not complete. This gives us a strong motivation to study these kinds of polynomials. In this paper, we are interested in developing some new formulas of the sixth-kind Chebyshev polynomials. To the best of our knowledge, the following formulas are not found in the literature:

- The connection formulas of Chebyshev polynomials of the sixth-kind with other orthogonal polynomials and their inversion ones.

- The moment formulas of Chebyshev polynomials of the sixth-kind.

- The linearization formulas of Chebyshev polynomials of the sixth-kind.

The importance of the above formulas of course gives a motivation to establish them.

In this article, to solve the connection problems between the sixth-kind Chebyshev polynomials and some other orthogonal polynomials, we follow two different approaches. Furthermore, and in order to establish the linearization formulas of Chebyshev polynomials of the sixth-kind, we first develop the moment formulas of these polynomials and then utilize them to establish the linearization formulas.

The arrangement of the paper is as follows. In the next section, some elementary properties and useful formulas of the sixth-kind Chebyshev polynomials are presented. Section 3 is concerned with the solutions to the connection problems between the sixth-kind Chebyshev polynomials and some other orthogonal polynomials and their inversion ones. New moment formulas of the sixth-kind Chebyshev polynomials are established in Sect. 4. Based on these formulas, the linearization formulas of the sixth-kind Chebyshev polynomials are derived in Sect. 5. We end the paper by presenting some conclusions in Sect. 6 .

\section{Some properties and essential formulas}

This section is devoted to presenting some fundamental properties of the sixth-kind Chebyshev polynomials. These polynomials satisfy the following orthogonality relation (see [6]):

$$
\int_{-1}^{1} x^{2} \sqrt{1-x^{2}} Y_{i}(x) Y_{j}(x) d x= \begin{cases}h_{i}, & \text { if } i=j \\ 0, & \text { if } i \neq j,\end{cases}
$$


with

$$
h_{i}=\frac{\pi}{2^{2 i+3}} \begin{cases}1, & i \text { even } \\ \frac{i+3}{i+1}, & i \text { odd }\end{cases}
$$

They also can be generated with the aid of the recurrence relation:

$$
\begin{aligned}
& Y_{j}(x)=x Y_{j-1}(x)-\frac{j(j+1)+(-1)^{j}(2 j+1)+1}{4 j(j+1)} Y_{j-2}(x), \\
& Y_{0}(x)=1, \quad Y_{1}(x)=x, \quad j \geq 2 .
\end{aligned}
$$

These polynomials can be expressed in the following power form representations (see [5]):

$$
\begin{aligned}
& Y_{2 n}(x)=\frac{\Gamma\left(\frac{3}{2}+n\right)}{(2 n+1) !} \sum_{k=0}^{n} \frac{(-1)^{k}\left(\begin{array}{c}
n \\
n-k
\end{array}\right)(1+2 n-k) !}{\Gamma\left(\frac{3}{2}+n-k\right)} x^{2 n-2 k}, \\
& Y_{2 n+1}(x)=\frac{\Gamma\left(n+\frac{5}{2}\right)}{(2 n+2) !} \sum_{k=0}^{n} \frac{(-1)^{k}\left(\begin{array}{c}
n \\
n-k
\end{array}\right)(2+2 n-k) !}{\Gamma\left(n-k+\frac{5}{2}\right)} x^{2 n-2 k+1} .
\end{aligned}
$$

The inversion formula to the power form representation of any polynomial is among the essential formulas associated with any set of polynomials. In the following lemma, we give the inversion formulas to the power form representations in (3) and (4).

Lemma 1 Let $n$ be any non-negative integer. The inversion formulas to the power form representations in (3) and (4) are given as follows:

$$
\begin{aligned}
& x^{2 n}=(2 n+1) ! \sum_{k=0}^{n} \frac{2^{1-2 k}(1-k+n)}{k !(2 n-k+2) !} Y_{2 n-2 k}(x), \\
& x^{2 n+1}=(3+2 n)(2 n+1) ! \sum_{k=0}^{n} \frac{2^{1-2 k}(1-k+n)}{k !(2 n-k+3) !} Y_{2 n-2 k+1}(x) .
\end{aligned}
$$

Proof The two inversion formulas in (5) and (6) can be written in the form

$$
x^{n}=\left\lfloor\frac{n}{2}\right\rfloor !\left(\frac{3}{2}\right)_{\left\lfloor\frac{1+n}{2}\right\rfloor} \sum_{k=0}^{\left\lfloor\frac{n}{2}\right\rfloor} \frac{(n-2 k+2) !}{k !\left\lfloor\frac{1}{2}(n-2 k)\right\rfloor !(n-k+2) !\left(\frac{3}{2}\right)_{\left\lfloor\frac{1}{2}(1+n-2 k)\right\rfloor}} Y_{n-2 k}(x),
$$

where $(u)_{m}$ represents the well-known Pochhammer symbol, that is, $(u)_{m}=\frac{\Gamma(u+m)}{\Gamma(u)}$, and $\lfloor z\rfloor$ denotes the greatest integer less than or equal to $z$.

With the aid of the recurrence relation (2), Eq. (7) can be proved through induction.

The next two lemmas are helpful in the sequel. A trigonometric representation of the polynomials $Y_{n}(x)$ is given in the first lemma. In contrast, the second lemma is concerned with the definition of $Y_{j}(x)$ for all $j \in \mathbb{Z}$. 
Lemma 2 ([7]) Let $n$ be any integer. We have

$$
Y_{n}(\cos \theta)= \begin{cases}\frac{\sin ((n+2) \theta)}{2^{n} \sin (2 \theta)}, & \text { n even }, \\ \frac{\sin ((n+1) \theta)+(n+1) \cos (\theta) \sin ((n+2) \theta)}{2^{n+1}(n+1) \cos ^{2}(\theta) \sin (\theta)}, & \text { n odd } .\end{cases}
$$

Lemma 3 For $n \leq-3$, we have

$$
Y_{n}(x)=\frac{-1}{2^{2 n+4}} Y_{-n-4}(x) \begin{cases}1, & n \text { even } \\ \frac{n+3}{n+1}, & n \text { odd }\end{cases}
$$

Proof The identity (9) is a direct consequence of the trigonometric identity (8).

\section{Connection formulas between the sixth-kind Chebyshev polynomials and some orthogonal polynomials}

This section deals with the connection problems between the sixth-kind Chebyshev polynomials and some other orthogonal polynomials. We will show that the connection coefficients can be expressed in terms of hypergeometric functions of certain arguments. Furthermore, we will show that in some cases they can be reduced to obtain some connection formulas whose coefficients are free of any hypergeometric functions.

\subsection{Solution of the sixth-kind Chebyshev-ultraspherical connection problem}

Theorem 1 Let $k$ be any non-negative integer. The sixth-kind Chebyshev-ultraspherical connection formula is

$$
Y_{k}(x)=\sum_{m=0}^{k} A_{m, k, \mu} C_{k-2 m}^{(\mu)}(x)
$$

with

$$
\begin{aligned}
& A_{m, k, \mu}=\frac{2^{1-k-2 \mu} \sqrt{\pi}(k-2 m+\mu) k ! \Gamma(k-2 m+2 \mu)}{(k-2 m) ! m ! \Gamma\left(\frac{1}{2}+\mu\right) \Gamma(1+k-m+\mu)} \\
& \times\left\{\begin{array}{cc}
{ }_{3} F_{2}\left(\begin{array}{cc}
-\frac{1}{2}-\frac{k}{2},-m,-k+m-\mu \\
-1-k, \frac{1}{2}-\frac{k}{2}
\end{array} \mid 1\right), & \text { k even }, \\
{ }_{3} F_{2}\left(\begin{array}{cc}
-1-\frac{k}{2},-m,-k+m-\mu \\
-1-k,-\frac{k}{2}
\end{array} \mid 1\right), & k \text { odd } .
\end{array}\right.
\end{aligned}
$$

Proof The connection formula (10) can be split into the following two connection formulas:

$$
\begin{aligned}
Y_{2 k}(x)= & \frac{\sqrt{\pi} 2^{1-2 k-2 \mu}(2 k) !}{\Gamma\left(\frac{1}{2}+\mu\right)} \sum_{m=0}^{k} \frac{(2 k-2 m+\mu) \Gamma(2(k-m+\mu))}{(2 k-2 m) ! m ! \Gamma(1+2 k-m+\mu)} \\
& \times{ }_{3} F_{2}\left(\begin{array}{c}
-m,-k-\frac{1}{2},-2 k-\mu+m \\
-2 k-1, \frac{1}{2}-k
\end{array} \mid 1\right) C_{2 k-2 m}^{(\mu)}(x)
\end{aligned}
$$


and

$$
\begin{aligned}
Y_{2 k+1}(x)= & \frac{2^{-2(k+\mu)} \sqrt{\pi}(1+2 k) !}{\Gamma\left(\frac{1}{2}+\mu\right)} \sum_{m=0}^{k} \frac{(1+2 k-2 m+\mu) \Gamma(1+2 k-2 m+2 \mu)}{m !(1+2 k-2 m) ! \Gamma(2+2 k-m+\mu)} \\
& \times{ }_{3} F_{2}\left(\begin{array}{c}
-m,-\frac{3}{2}-k,-1-2 k+m-\mu \\
-2-2 k,-\frac{1}{2}-k
\end{array} \mid 1\right) C_{2 k-2 m+1}^{(\mu)}(x) .
\end{aligned}
$$

We prove (12). The proof of (11) is similar. Now, consider the following connection formula:

$$
Y_{2 k+1}(x)=\sum_{m=0}^{k} \xi_{m, k, \mu} C_{2 k-2 m+1}^{(\mu)}(x)
$$

where $\xi_{m, k, \mu}$ are the connection coefficients to be determined. We make use of the orthogonality relation of ultraspherical polynomials ([25]) to get the integral form of these coefficients,

$$
\begin{aligned}
\xi_{k, m, \mu}= & \frac{2^{1-2 \mu}(1+2 k-2 m+\mu) \Gamma(1+2 k-2 m+2 \mu)}{(2 k-2 m+1) !\left(\Gamma\left(\frac{1}{2}+\mu\right)\right)^{2}} \\
& \times \int_{-1}^{1}\left(1-x^{2}\right)^{\mu-\frac{1}{2}} Y_{2 k+1}(x) C_{2 k-2 m+1}^{(\mu)}(x) d x .
\end{aligned}
$$

In virtue of Rodrigues' formula of $C_{j}^{(\mu)}(x)$ (see [25]), Eq. (13) turns into the form

$$
\begin{aligned}
\xi_{k, m, \mu}= & \frac{-4^{-k+m-\mu}(1+2 k-2 m+\mu) \Gamma(1+2 k-2 m+2 \mu)}{(2 k-2 m+1) ! \Gamma\left(\frac{1}{2}+\mu\right) \Gamma\left(\frac{3}{2}+2 k-2 m+\mu\right)} \\
& \times \int_{-1}^{1} Y_{2 k+1}(x) D^{2 k-2 m+1}\left(1-x^{2}\right)^{2 k-2 m+\mu+\frac{1}{2}} d x .
\end{aligned}
$$

Integrating the right-hand side of the last equation by parts $(2 k-2 m+1)$ times enables one to rewrite (14) as

$$
\begin{aligned}
\xi_{k, m, \mu}= & \frac{4^{-k+m-\mu}(1+2 k-2 m+\mu) \Gamma(1+2 k-2 m+2 \mu)}{(2 k-2 m+1) ! \Gamma\left(\frac{1}{2}+\mu\right) \Gamma\left(\frac{3}{2}+2 k-2 m+\mu\right)} \\
& \times \int_{-1}^{1}\left(1-x^{2}\right)^{2 k-2 m+\mu+\frac{1}{2}} D^{2 k-2 m+1} Y_{2 k+1}(x) d x
\end{aligned}
$$

Making use of the power form representation of $Y_{2 k+1}(x)$ in (4) transforms (15) into the following form:

$$
\begin{aligned}
\xi_{k, m, \mu}= & \frac{\left(\frac{3}{2}+k\right)(1+2 k-2 m+\mu) \Gamma(1+2 k-2 m+2 \mu)}{(1+k)(2 k-2 m+1) ! \Gamma\left(\frac{1}{2}+\mu\right) \Gamma\left(\frac{3}{2}+2 k-2 m+\mu\right)} \\
& \times \sum_{j=0}^{k} \frac{(-1)^{j} 2^{-2(k-m+j+\mu)}(2 k-j+2) !}{(3+2 k-2 j) j !(2 m-2 j) !} \int_{-1}^{1}\left(1-x^{2}\right)^{2 k-2 m+\mu+\frac{1}{2}} x^{2 m-2 j} d x .
\end{aligned}
$$


If we note the identity

$$
\int_{-1}^{1}\left(1-x^{2}\right)^{2 k-2 m+\mu+\frac{1}{2}} x^{2 m-2 j} d x=\frac{\Gamma\left(\frac{1}{2}+m-j\right) \Gamma\left(\frac{3}{2}+2 k-2 m+\mu\right)}{\Gamma(2+2 k-m-j+\mu)},
$$

then the coefficients $\xi_{k, m, \mu}$ are given by

$$
\begin{aligned}
\xi_{k, m, \mu}= & \frac{\sqrt{\pi} 2^{-2(k+\mu)}\left(\frac{3}{2}+k\right)(1+2 k-2 m+\mu) \Gamma(1+2 k-2 m+2 \mu)}{(1+k)(2 k-2 m+1) ! \Gamma\left(\frac{1}{2}+\mu\right)} \\
& \times \sum_{j=0}^{k} \frac{(-1)^{j}(2 k-j+2) !}{(3+2 k-2 j) j !(m-j) ! \Gamma(2+2 k-m-j+\mu)} .
\end{aligned}
$$

The summation in (16) can be written in a hypergeometric form as

$$
\begin{aligned}
& \sum_{j=0}^{k} \frac{(-1)^{j}(2 k-j+2) !}{(3+2 k-2 j) j !(m-j) ! \Gamma(2+2 k-m-j+\mu)} \\
& \quad=\frac{(2 k+2) !}{(3+2 k) m ! \Gamma(2+2 k-m+\mu)}{ }_{3} F_{2}\left(\begin{array}{c}
-m,-\frac{3}{2}-k,-1-2 k+m-\mu \\
-2-2 k,-\frac{1}{2}-k
\end{array} \mid 1\right),
\end{aligned}
$$

and therefore, the following connection formula is obtained:

$$
\begin{aligned}
Y_{2 k+1}(x)= & \frac{2^{-2(k+\mu)} \sqrt{\pi}(1+2 k) !}{\Gamma\left(\frac{1}{2}+\mu\right)} \sum_{m=0}^{k} \frac{(1+2 k-2 m+\mu) \Gamma(1+2 k-2 m+2 \mu)}{m !(1+2 k-2 m) ! \Gamma(2+2 k-m+\mu)} \\
& \times{ }_{3} F_{2}\left(\begin{array}{c}
-m,-\frac{3}{2}-k,-1-2 k+m-\mu \\
-2-2 k,-\frac{1}{2}-k
\end{array} \mid 1\right) C_{2 k-2 m+1}^{(\mu)}(x) .
\end{aligned}
$$

Noting that Chebyshev polynomials of the first- and second-kinds and Legendre polynomials are special ones of the ultraspherical polynomials, the connection formulas between the sixth-kind Chebyshev polynomials and these polynomials can be deduced as special cases of (11) and (12). These results are given in the following corollaries.

Corollary 1 Let $k$ be any non-negative integer. The sixth-and first-kind Chebyshev polynomials are connected by the following two formulas:

$$
\begin{aligned}
& Y_{2 k}(x)=\frac{1}{2^{2 k-1}} \sum_{m=0}^{\left\lfloor\frac{k}{2}\right\rfloor} T_{2 k-4 m}(x), \\
& Y_{2 k+1}(x)=\frac{1}{(k+1) 2^{2 k}}\left(\sum_{m=0}^{\left\lfloor\frac{k}{2}\right\rfloor}(1+k-m) T_{2 k-4 m+1}(x)+\sum_{m=0}^{\left\lfloor\frac{k-1}{2}\right\rfloor}(1+m) T_{2 k-4 m-1}(x)\right) .
\end{aligned}
$$

Proof We prove (17). Setting $\mu=0$ in Eq. (12) yields

$$
\begin{aligned}
Y_{2 k+1}(x)= & \frac{(1+2 k) !}{2^{2 k}} \sum_{m=0}^{k} \frac{1}{m !(2 k-m+1) !} \\
& \times{ }_{3} F_{2}\left(\begin{array}{c}
-m,-\frac{3}{2}-k,-1-2 k+m \\
-2-2 k,-\frac{1}{2}-k
\end{array} \mid 1\right) T_{2 k-2 m+1}(x) .
\end{aligned}
$$


Based on Zeilberger's algorithm (see Koepf [26]), and if we set

$$
G_{m, k}={ }_{3} F_{2}\left(\begin{array}{c}
-m,-\frac{3}{2}-k,-1-2 k+m \\
-2-2 k,-\frac{1}{2}-k
\end{array} \mid 1\right)
$$

then $G_{m, k}$ satisfies the following recurrence relation of order two:

$$
\begin{aligned}
& m(1-m)(-1-k+m) G_{m-2, k}-m(-3-2 k+m) G_{m-1, k} \\
& +(-3-2 k+m)(-2-2 k+m)(-2-k+m) G_{m, k}=0 \\
& G_{0, k}=1, \quad G_{1, k}=\frac{1}{(k+1)(2 k+1)} .
\end{aligned}
$$

The last recurrence relations can be exactly solved to give

$$
G_{m, k}= \begin{cases}\frac{m !(2 k-m+2) !}{(2 k+2) !}, & m \text { even } \\ \frac{(m+1) !(2 k-m+1) !}{(2 k+2) !}, & m \text { odd }\end{cases}
$$

and therefore, the connection formula (17) can be obtained.

Corollary 2 Let $k$ be any non-negative integer. The sixth-and second-kind Chebyshev polynomials are connected by the following two formulas:

$$
\begin{aligned}
& Y_{2 k}(x)=\frac{1}{2^{2 k}} \sum_{m=0}^{k}(-1)^{m} U_{2 k-2 m}(x), \\
& Y_{2 k+1}(x)=\frac{1}{(k+1) 2^{2 k+1}} \sum_{m=0}^{k}(-1)^{m}(k-m+1) U_{2 k-2 m+1}(x) .
\end{aligned}
$$

Proof

We prove (18). Setting $\mu=1$ in the connection formula (11) yields

$$
\begin{aligned}
Y_{2 k}(x)= & \frac{(2 k+1) !}{2^{2 k}} \sum_{m=0}^{k} \frac{1+k-m}{(2 k-m+2) ! m !} \\
& \times{ }_{3} F_{2}\left(\begin{array}{c}
-m,-\frac{3}{2}-k,-2-2 k+m \\
-2-2 k,-\frac{1}{2}-k
\end{array} \mid 1\right) U_{2 k-2 m+1}(x) .
\end{aligned}
$$

The Pfaff-Saalschütz identity ([27]) reduces the above hypergeometric function to the form

$$
{ }_{3} F_{2}\left(\begin{array}{c}
-m,-\frac{3}{2}-k,-2-2 k+m \\
-2-2 k,-\frac{1}{2}-k
\end{array} \mid 1\right)=\frac{(-1)^{m} m !(2 k-m+2) !}{(2 k+2) !}
$$

and therefore, the following connection formula is obtained:

$$
Y_{2 k+1}(x)=\frac{1}{(k+1) 2^{2 k+1}} \sum_{m=0}^{k}(-1)^{m}(k-m+1) U_{2 k-2 m+1}(x) .
$$


Corollary 3 Let $k$ be any non-negative integer. The sixth-kind Chebyshev polynomials and Legendre polynomials are connected by the following two formulas:

$$
\begin{aligned}
Y_{2 k}(x)= & \frac{\sqrt{\pi}(2 k) !}{2^{2 k+1}} \sum_{m=0}^{k} \frac{1+4 k-4 m}{m ! \Gamma\left(\frac{3}{2}+2 k-m\right)} \\
& \times{ }_{3} F_{2}\left(\begin{array}{c}
-m,-k-\frac{1}{2},-2 k+m-\frac{1}{2} \\
-2 k-1, \frac{1}{2}-k
\end{array} \mid 1\right) P_{2 k-2 m}(x), \\
Y_{2 k+1}(x)= & \frac{\sqrt{\pi}(2 k+1) !}{2^{2 k+2}} \sum_{m=0}^{k} \frac{3+4 k-4 m}{m ! \Gamma\left(\frac{5}{2}+2 k-m\right)} \\
& \times{ }_{3} F_{2}\left(\begin{array}{c}
-m,-\frac{3}{2}-k,-\frac{3}{2}-2 k+m \\
-2-2 k,-\frac{1}{2}-k
\end{array} \mid 1\right) P_{2 k-2 m+1}(x) .
\end{aligned}
$$

Proof The two connection formulas (19) and (20) can be immediately concluded by setting $\mu=\frac{1}{2}$, in (11) and (12), respectively.

\subsection{Solution of the ultraspherical sixth-kind Chebyshev connection problem}

This section derives the inversion connection formulas to those given in the last subsection.

Theorem 2 Let $k$ be any non-negative integer. The ultraspherical sixth-kind Chebyshev polynomial connection formula is

$$
C_{k}^{(\mu)}(x)=\sum_{m=0}^{k} H_{m, k, \mu} Y_{k-2 m}(x),
$$

with

$$
\begin{aligned}
H_{m, k, \mu}= & \frac{2^{-1+k-2 m+2 \mu} k ! \Gamma\left(\frac{1}{2}+\mu\right) \Gamma(k-m+\mu) \Gamma(-2+m+\mu)}{\sqrt{\pi} m !(2+k-m) ! \Gamma(-1+\mu) \Gamma(k+2 \mu)} \\
& \times \begin{cases}(2+k-2 m)\left(-2-2 k+4 m+2 k m-2 m^{2}+\mu+k \mu\right), & k \text { even }, \\
(1+k-2 m)\left(-4-2 k+4 m+2 k m-2 m^{2}+2 \mu+k \mu\right), & k \text { odd } .\end{cases}
\end{aligned}
$$

Proof The connection formula (21) can be split into the following two connection formulas:

$$
\begin{aligned}
C_{2 k}^{(\mu)}(x)= & \frac{(2 k) ! \Gamma\left(\frac{1}{2}+\mu\right)}{\sqrt{\pi} \Gamma(-1+\mu) \Gamma(2(k+\mu)} \sum_{m=0}^{k} \frac{4^{k-m+\mu}(1+k-m) \Gamma(2 k-m+\mu)}{m !(2 k-m+2) !} \\
& \times \Gamma(-2+m+\mu)\left\{-2(-1+m)^{2}+\mu+2 k(-2+2 m+\mu)\right\} Y_{2 k-2 m}(x), \\
C_{2 k+1}^{(\mu)}(x)= & \frac{(1+2 k) ! \Gamma\left(\frac{5}{2}+k\right) \Gamma\left(\frac{1}{2}+\mu\right) \Gamma(1+2 k+\mu)}{\sqrt{\pi}(3+2 k)(2 k+\mu) \Gamma\left(\frac{3}{2}+k\right) \Gamma(1+2 k+2 \mu)} \\
& \times \sum_{m=0}^{k} \frac{2^{2(1+k-m+\mu)}(1+k-m)(\mu-1)_{m-1}}{(2 k-m+3) ! m !(1+2 k-m+\mu)_{m-1}} \\
& \times\left\{\left(-6+6 m-2 m^{2}+3 \mu+2 k(-2+2 m+\mu)\right\} Y_{2 k-2 m+1}(x) .\right.
\end{aligned}
$$


We prove (22). The power form representation of ultraspherical polynomials enables one to write

$$
C_{2 k}^{(\mu)}(x)=\frac{(2 k) ! \Gamma(1+2 \mu)}{\Gamma(1+\mu) \Gamma(2(k+\mu))} \sum_{r=0}^{k} \frac{(-1)^{r} 2^{-1+2 k-2 r} \Gamma(2 k-r+\mu)}{r !(2 k-2 r) !} x^{2 k-2 r} .
$$

With the aid of Eq. (5), Eq. (24) can be converted into

$$
\begin{aligned}
C_{2 k}^{(\mu)}(x)= & \frac{(2 k) ! \Gamma(1+2 \mu)}{\Gamma(1+\mu) \Gamma(2(k+\mu))} \sum_{r=0}^{k} \frac{(-1)^{r} 2^{-1+2 k-2 r}(1+2 k-2 r) \Gamma(2 k-r+\mu)}{r !} \\
& \times \sum_{m=0}^{k-r} \frac{2^{1-2 m}(k-m-r+1)}{m !(2 k-m-2 r+2) !} Y_{2 k-2 m-2 r}(x) .
\end{aligned}
$$

Carrying out certain algebraic computations on the last formula transforms it into the following form:

$$
\begin{aligned}
C_{2 k}^{(\mu)}(x)= & \frac{(2 k) ! \Gamma\left(\frac{1}{2}+\mu\right)}{\sqrt{\pi} \Gamma(2(k+\mu))} \sum_{m=0}^{k} 4^{k-m+\mu}(1+k-m) \\
& \times \sum_{p=0}^{m} \frac{(-1)^{p}(1+2 k-2 p) \Gamma(2 k-p+\mu)}{p !(m-p) !(2 k-m-p+2) !} Y_{2 k-2 m}(x),
\end{aligned}
$$

and therefore the following connection formula is obtained:

$$
\begin{aligned}
C_{2 k}^{(\mu)}(x)= & \frac{2^{-1+2 \mu}(2 k) ! \Gamma\left(\frac{1}{2}+\mu\right) \Gamma(2 k+\mu)}{\left(\frac{1}{2}+k\right) \Gamma(2(k+\mu))} \sum_{m=0}^{k} \frac{(-1)^{m}\left(\frac{3}{2}+k-m\right)_{m}}{(k-m) ! m ! \Gamma(-2-2 k+m)_{m}} \\
& \times{ }_{3} F_{2}\left(\begin{array}{c}
-m, \frac{1}{2}-k,-2-2 k+m \\
-\frac{1}{2}-k, 1-2 k-\mu
\end{array} \mid 1\right) Y_{2 k-2 m}(x) .
\end{aligned}
$$

Based on symbolic computation, and in particular, on Zeilberger's and Petkovsek's algorithms (see Koepf [26]), it can be shown that

$$
\begin{aligned}
{ }_{3} F_{2} & \left(\begin{array}{c}
-m, \frac{1}{2}-k,-2 k+m-2 \\
-k-\frac{1}{2},-2 k-\mu+1
\end{array} \mid 1\right) \\
& =\frac{(\mu-1)_{m-1} \Gamma(2 k-m+\mu)}{(2 k+1) \Gamma(2 k+\mu)}\left\{-2(-1+m)^{2}+\mu+2 k(-2+2 m+\mu)\right\},
\end{aligned}
$$

then after performing some calculations, the connection formula (22) can be obtained. Equation (23) can be similarly proved.

Now, setting $\mu=0,1$ and $\frac{1}{2}$, respectively, in the connection formulas (22) and (23), and performing some reductions yield three special connection formulas. The results are given in the following corollaries. 
Corollary 4 Let $k$ be any non-negative integer. The first-kind and sixth-kind Chebyshev polynomials connection formulas are

$$
\begin{aligned}
& T_{2 k}(x)=2^{2 k-5}\left\{16 Y_{2 k}(x)-Y_{2 k-4}(x)\right\}, \\
& T_{2 k+1}(x)=4^{k-2}\left(16 Y_{2 k+1}(1)-\frac{4}{1+k} Y_{2 k-1}(x)+\frac{1-k}{k} Y_{2 k-3}(x)\right) .
\end{aligned}
$$

Corollary 5 Let $k$ be any non-negative integer. The second-kind and sixth-kind Chebyshev polynomials connection formulas are

$$
\begin{aligned}
& U_{2 k}(x)=2^{2 k-2}\left\{4 Y_{2 k}(x)+Y_{2 k-2}(x)\right\}, \\
& U_{2 k+1}(x)=2^{2 k-1}\left(4 Y_{2 k+1}+\frac{k}{1+k} Y_{2 k-1}(x)\right) .
\end{aligned}
$$

Corollary 6 Let $k$ be any non-negative integer. The Legendre polynomials and sixth-kind Chebyshev polynomials connection formulas are

$$
\begin{aligned}
P_{2 k}(x)= & \frac{1}{\pi} \sum_{m=0}^{k} \frac{2^{-1+2 k-2 m}(1+k-m) \Gamma\left(\frac{1}{2}+2 k-m\right) \Gamma\left(-\frac{3}{2}+m\right)}{m !(2 k-m+2) !} \\
& \times\{3+4(-2+m) m+2 k(3-4 m)\} Y_{2 k-2 m}(x), \\
P_{2 k+1}(x)= & \frac{1}{\pi} \sum_{m=0}^{k} \frac{4^{k-m}(1+k-m) \Gamma\left(\frac{3}{2}+2 k-m\right) \Gamma\left(-\frac{3}{2}+m\right)}{m !(2 k-m+3) !} \\
& \times\left\{(3-2 m)^{2}+2 k(3-4 m)\right\} Y_{2 k-2 m+1}(x) .
\end{aligned}
$$

\section{Development of the moment formulas of the sixth-kind Chebyshev polynomials}

This section focuses on the development of the moment formulas of Chebyshev polynomials of the sixth-kind. First, the following lemma serves in the proof of the main theorem of the moment formulas.

Lemma 4 Let $\ell, j$ and $m$ be any non-negative integers. The following reduction formula holds:

$$
\begin{aligned}
&{ }_{4} F_{3}\left(\begin{array}{c}
-i,-k-\frac{3}{2},-k, i-2 k-2 r-4 \\
-2 k-2,-k-r-\frac{3}{2},-k-r-1
\end{array} \mid 1\right) \\
&=\frac{(2 r) !}{2(1+k)(2-i+k+r)(3+2 k+2 r) !} \\
& \times \begin{cases}\frac{(1+i+k+2(1+k) r)(-i+2(2+k+r)) !}{(1-i+2 r) !}, & 0 \leq i \leq k, \\
\frac{(-5+i-3 k-2(2+k) r) !}{(i-2 k-3) !}+\frac{(1+i+k+2(1+k) r)(-i+2(2+k+r)) !}{(-i+2 r+1) !}, & i \geq k+1 .\end{cases}
\end{aligned}
$$

Proof Let

$$
A_{i, r, k}={ }_{4} F_{3}\left(\begin{array}{c}
-i,-k,-k-\frac{3}{2}, i-2 k-2 r-4 \\
-2 k-2,-k-r-\frac{3}{2},-k-r-1
\end{array} \mid 1\right) .
$$


First, since the terminating hypergeometric function in (25) involves two non-negative integers $i$ and $k$, we have to take into consideration the following two cases:

(i) For $i \leq k$, Zeilberger's algorithm enables one to obtain the following recurrence relation that is satisfied by $A_{i, r, k}$ :

$$
\begin{aligned}
& (i-1)(-3+i-2 r)(-4-k+i-r) A_{i-2, r, k} \\
& \quad+2\left(i^{2}-2 i k-2 i r+2 k r-6 i+3 k+4 r+7\right)(3+k+r-i) A_{i-1, r, k} \\
& \quad+(-5-2 k+i-2 r)(-3-2 k+i)(-2-k-r+i) A_{i, r, k}=0,
\end{aligned}
$$

with the following initial values:

$$
A_{0, r, k}=1, \quad A_{1, r, k}=\frac{2+k+2 r+2 k r}{2(1+k)(1+k+r)} .
$$

The recurrence relation (26) can be exactly solved to give

$$
A_{i, r, k}=\frac{(2 r) !(1+i+k+2(1+k) r)(-i+2(2+k+r)) !}{2(1+k)(2-i+k+r)(1-i+2 r) !(3+2 k+2 r) !}
$$

(ii) For $i \geq k+1$, Zeilberger's algorithm again enables one to obtain the following recurrence relation that is satisfied by $A_{i, r, k}$ :

$$
\begin{aligned}
& (k-1)(k+1)(i-2 k+1)(i-2 k)(-1-2 k+i-2 r)(-2-2 k+i-2 r) \\
& \quad \times(-r-k+i)\left(2 i k-4 k^{2}-2 k r+2 i-9 k-2 r-4\right) A_{i, r, k-2}-4 k(1+2 k+2 r) \\
& \quad \times(k+r)(-k-1+i-r)\left(2 i^{3} k^{2}-12 i^{2} k^{3}-6 i^{2} k^{2} r+24 i k^{4}+24 i k^{3} r+8 i k^{2} r^{2}\right. \\
& \quad-16 k^{5}-24 k^{4} r-16 k^{3} r^{2}-4 k^{2} r^{3}+2 i^{3} k-21 i^{2} k^{2}-6 i^{2} k r+60 i k^{3}+46 i k^{2} r \\
& +8 i k r^{2}-52 k^{4}-68 k^{3} r-32 k^{2} r^{2}-4 k r^{3}+i^{3}-10 i^{2} k-3 i^{2} r+41 i k^{2}+24 i k r \\
& +2 i r^{2}-50 k^{3}-55 k^{2} r-14 k r^{2}-2 i^{2}+6 i k+2 i r-10 k^{2}-6 k r+2 r^{2}-i \\
& +6 k+5 r+2) A_{i, r, k-1}+4 k(1+k)(1+k+r)(k+r)(3+2 k+2 r) \\
& \quad \times(1+2 k+2 r)\left(2 i k-4 k^{2}-2 k r-k+1\right)(-2-k-r+i) A_{i, r, k}=0
\end{aligned}
$$

with the initial values

$$
A_{i, r, 0}=1, \quad A_{i, r, 1}=1+\frac{5 i(i-2(r+3))}{4(r+2)(2 r+5)}
$$

The recurrence relation (27) can be exactly solved to give

$$
\begin{aligned}
A_{i, r, k}= & \frac{i !(2 r) !(-i+2(2+k+r)) !}{2(1+k)(2-i+k+r)(3+2 k+2 r) !} \\
& \times\left\{\frac{1+i+k+2(1+k) r}{i !(1-i+2 r) !}+\frac{-5+i-3 k-2(2+k) r}{(-3+i-2 k) !(-i+2(2+k+r)) !}\right\} .
\end{aligned}
$$

Lemma 4 is now proved. 
Theorem 3 The following moment formula holds for all non-negative integers $r$ and $k$ :

$$
x^{r} Y_{k}(x)=\sum_{i=0}^{r} B_{i, r, k} Y_{r+k-2 i}(x)
$$

where the coefficients $B_{i, r, k}$ are given explicitly as

$$
\begin{aligned}
B_{i, r, k}= & \frac{\left(\begin{array}{l}
r \\
2
\end{array}\right)}{2^{2 i}} \\
& \times \begin{cases}1, & r, k \text { even }, \\
\frac{-4 i^{2}+(1+k)(-1+r)(3+k+r)+2 i(2+k-k r)}{(1+k)(-1+r)(3-2 i+k+r)}, & r \text { even }, k \text { odd }, \\
\frac{r(3+k+r)-2 i(1+r)}{r(3-2 i+k+r)}, & r \text { odd }, k \text { even }, \\
\frac{2 i+r+k r}{(1+k) r}, & r, k \text { odd } .\end{cases}
\end{aligned}
$$

Proof The proof of the theorem is lengthy. The idea depends on proving its alternative form. In fact, the moments formula (28) with the coefficients in (29) can be split into the following four moment formulas:

$$
\begin{aligned}
x^{2 r} Y_{2 k}(x)=(2 r) ! & \sum_{i=0}^{2 r} \frac{1}{2^{2 i} i !(2 r-i) !} Y_{2 k+2 r-2 i}(x), \\
x^{2 r} Y_{2 k+1}(x)= & \frac{(2 r) !}{(2 r-1)(1+k)} \sum_{i=0}^{2 r} \frac{1}{2^{2 i+1}(2+k-i+r) i !(2 r-i) !} \\
& \times\left\{-4+3 i+6 r-2 k(-3+i-r)(-1+2 r)-2(i-r)(i+2 r)-2 k^{2}(1-2 r)\right\} \\
& \times Y_{2 k+2 r-2 i+1}(x), \\
x^{2 r+1} Y_{2 k}(x)= & (2 r) ! \sum_{i=0}^{2 r+1} \frac{k+2 k r-2 i(1+r)+(2+r)(1+2 r)}{2^{2 i} i !(2+k-i+r)(1-i+2 r) !} Y_{2 k+2 r-2 i+1}(x), \\
x^{2 r+1} Y_{2 k+1}(x)= & \frac{(2 r) !}{k+1} \sum_{i=0}^{2 r+1} \frac{1+k+i+2(1+k) r}{2^{2 i} i !(2 r-i+1) !} Y_{2 k+2 r-2 i+2}(x) .
\end{aligned}
$$

Now, we are going to prove the moment formula (30). The remaining moment formulas can be proved similarly. The power form expression in (4) along with the inversion formula in (5) enables one to write

$$
\begin{aligned}
x^{2 r+1} Y_{2 k+1}(x)= & \frac{3+2 k}{4(1+k)} \sum_{i=0}^{k+r} \frac{(-1)^{i}(-i+2 k+2) !(-2 i+2 k+2 r+3) !}{2^{2 i-1}(3-2 i+2 k) i !(-2 i+2 k+1) !} \\
& \times \sum_{\ell=0}^{k+r-i+1} \frac{(k-\ell+r-i+2)}{2^{2 \ell-1} \ell !(2 k-\ell+2 r-2 i+4) !} Y_{2 k+2 r-2 \ell-2 i+2}(x) .
\end{aligned}
$$


Performing some computations on the last formula turns it into the formula

$$
\begin{aligned}
x^{2 r+1} Y_{2 k+1}(x)= & \frac{3+2 k}{1+k} \sum_{i=0}^{k+r+1} \frac{2+k-i+r}{2^{2 i}} \\
& \times\left(\sum_{p=0}^{i} \frac{(-1)^{p}(2 k-p+2) !(2 k+2 r-2 p+3) !}{(3+2 k-2 p)(2 k-2 p+1) ! p !(i-p) !(2 k-i+2 r-p+4) !}\right) \\
& \times Y_{2 k+2 r-2 i+2},
\end{aligned}
$$

which can be written alternatively as

$$
\begin{aligned}
x^{2 r+1} Y_{2 k+1}(x)= & (3+2 k+2 r) ! \sum_{i=0}^{k+r+1} \frac{(2+k-i+r)}{2^{2 i-1} i !(4+2 k-i+2 r) !} \\
& \times{ }_{4} F_{3}\left(\begin{array}{l}
-k,-i,-\frac{3}{2}-k,-4-2 k+i-2 r \\
-2-2 k,-\frac{3}{2}-k-r,-1-k-r
\end{array} \mid 1\right) Y_{2 k+2 r-2 i+2}(x) .
\end{aligned}
$$

A closed-form of the term ${ }_{4} F_{3}(1)$ that appears in (31) is given in Lemma 4, and accordingly this enables one to write

$$
x^{2 r+1} Y_{2 k+1}(x)=\sum_{i=0}^{r+k+1} B_{i, r, k} Y_{2 k+2 r-2 i+2}(x),
$$

where the moments' coefficients $B_{i, r, k}$ are given as

$$
\begin{aligned}
B_{i, r, k}= & \frac{(2 r) !}{2^{2 i}(k+1)} \\
& \times \begin{cases}\frac{(1+i+k+2(1+k) r)}{i !(-i+2 r+1) !}, & 0 \leq k \leq i, \\
\frac{-5+i-3 k-2(2+k) r}{(-i+2(2+k+r)) !(i-2 k-3) !}+\frac{1+i+k+2(1+k) r}{i !(-i+2 r+1) !}, & k+1 \leq i \leq k+r+1 .\end{cases}
\end{aligned}
$$

Due to Eq. (32) along with Eq. (33), we get

$$
\begin{aligned}
x^{2 r+1} Y_{2 k+1}(x)= & \frac{(2 r) !}{k+1} \sum_{i=0}^{r-k-2} 2^{-2(1+k-i+r)}\left\{\frac{2-i+3 r+2 k(1+r)}{(-k+i+r) !(k-i+r+1) !}\right. \\
& \left.-\frac{4+i+3 r+2 k(1+r)}{(-k-i+r-2) !(k+i+r+3) !}\right\} Y_{2 i}(x) \\
& +\sum_{i=r-k-1}^{r+k+1} \frac{2^{-2(1+k-i+r)}(2-i+3 r+2 k(1+r))}{(-k+i+r) !(k-i+r+1) !} Y_{2 i}(x),
\end{aligned}
$$

which can be written as

$$
\begin{aligned}
x^{2 r+1} Y_{2 k+1}(x)= & \frac{(2 r) !}{1+k}\left\{\sum_{i=0}^{k+r+1} \frac{2^{-2(1+k-i+r)}(2-i+3 r+2 k(1+r))}{(-k+i+r) !(k-i+r+1) !} Y_{2 i}(x)\right. \\
& \left.-\sum_{i=0}^{r-k-2} \frac{4^{-1-k+i-r}(4+i+3 r+2 k(1+r))}{(-k-i+r-2) !(k+i+r+3) !} Y_{2 i}(x)\right\} .
\end{aligned}
$$


Now, based on identity (9), we have

$$
Y_{-2 j}(x)=-2^{4 j-4} Y_{2 j-4}, \quad j \geq 1 .
$$

If we perform some suitable transformations on the second sum of (34), we can write

$$
\begin{aligned}
& \sum_{i=0}^{r-k-2} \frac{4^{-1-k+i-r}(4+i+3 r+2 k(1+r))}{(-k-i+r-2) !(k+i+r+3) !} Y_{2 i}(x) \\
& \quad=-\sum_{t=2}^{r-k} \frac{2^{-2(1+k+r+t)}(2+3 r+2 k(1+r)+t)}{(-k+r-t) !(k+r+t+1) !} Y_{-2 t}(x) \\
& \quad=-\sum_{i=k+r+2}^{2 r+1} \frac{2^{-2 i}(1+k+i+2(1+k) r)}{i !(1-i+2 r) !} Y_{2 k+2 r-2 i+2}(x),
\end{aligned}
$$

and accordingly, Eq. (34) can be rewritten in the form

$$
\begin{aligned}
x^{2 r+1} Y_{2 k+1}(x)= & \frac{(2 r) !}{1+k}\left\{\sum_{i=0}^{k+r+1} \frac{(1+k+i+2(1+k) r)}{2^{2 i} i !(1-i+2 r) !} Y_{2 k+2 r-2 i+2}(x)\right. \\
& \left.+\sum_{i=k+r+2}^{2 r+1} \frac{(1+k+i+2(1+k) r)}{2^{2 i} i !(1-i+2 r) !} Y_{2 k+2 r-2 i+2}(x)\right\},
\end{aligned}
$$

which is equivalent to

$$
x^{2 r+1} Y_{2 k+1}(x)=\frac{(2 r) !}{k+1} \sum_{i=0}^{2 r+1} \frac{1+k+i+2(1+k) r}{2^{2 i} i !(2 r-i+1) !} Y_{2 k+2 r-2 i+2}(x) .
$$

The proof is now complete.

\section{Linearization formulas of Chebyshev polynomials of the sixth-kind}

In this section, and based on the moment formulas introduced in Sect. 4, the linearization formulas of Chebyshev polynomials of the sixth-kind are established.

Theorem 4 For all non-negative integers $m$ and $n$, the following linearization formula is valid:

$$
Y_{m}(x) Y_{n}(x)=\sum_{r=0}^{m} L_{r, m, n} Y_{m+n-2 r}(x),
$$

and the linearization coefficients $L_{r, m, n}$ are given explicitly as

$$
\begin{aligned}
& L_{r, m, n}=\frac{1}{2^{2 r+1}} \\
& \times \begin{cases}1+(-1)^{r}, & \text { m, neven } \\
\frac{(2+n)(2+m+n-2 r)+(-1)^{r}\left(2+4 n+m n+n^{2}-2(2+m+n) r+2 r^{2}\right)}{(n+1)(3+m+n-2 r)}, & \text { m even, } n \text { odd }, \\
\frac{(2+m)(2+m+n-2 r)+(-1)^{r}\left(2+4 m+m^{2}+m n-2(2+m+n) r+2 r^{2}\right)}{(m+1)(3+m+n-2 r)}, & \text { modd, } n \text { even }, \\
\frac{(2+m)(2+n)+(-1)^{r}\left(-2+m n-2(2+m+n) r+2 r^{2}\right)}{(m+1)(n+1)}, & \text { modd, } n \text { odd } .\end{cases}
\end{aligned}
$$


Proof The proof of the theorem is lengthy. Equation (35) can be split into the following four relations:

$$
\begin{aligned}
Y_{2 m}(x) Y_{2 n}(x)=\sum_{r=0}^{m} & \frac{1}{2^{4 r}} Y_{2 m+2 n-4 r}(x), \\
Y_{2 m}(x) Y_{2 n+1}(x)= & \frac{1}{n+1} \sum_{r=0}^{2 m+1} \frac{1}{2^{2 r+3}(2+m+n-r)}\{(3+2 n)(3+2 m+2 n-2 r) \\
& \left.+(-1)^{r}\left(7+4 n^{2}+m(2+4 n-4 r)-4 n(-3+r)+2(-3+r) r\right)\right\} \\
& \times Y_{2 m+2 n-2 r+1}(x), \\
Y_{2 m+1}(x) Y_{2 n}(x)= & \frac{1}{m+1} \sum_{r=0}^{2 m+1} \frac{1}{2^{2 r+3}(2+m+n-r)}\{(3+2 m)(3+2 m+2 n-2 r) \\
& \left.+(-1)^{r}\left(7+2 n+4 m(3+m+n)-6 r-4(m+n) r+2 r^{2}\right)\right\} \\
& \times Y_{2 m+2 n-2 r+1}(x), \\
Y_{2 m+1}(x) Y_{2 n+1}(x)= & \frac{1}{(m+1)(n+1)} \sum_{r=0}^{2 m+1} \frac{1}{2^{2 r+3}}\{(3+2 m)(3+2 n) \\
& \left.+(-1)^{r}\left(-1+2 m+2 n+4 m n-4(2+m+n) r+2 r^{2}\right)\right\} \\
& \times Y_{2 m+2 n-2 r+2}(x) .
\end{aligned}
$$

Now, we prove formula (36). The other formulas can be proved similarly. Based on Eq. (4), we can write

$$
Y_{2 m+1}(x) Y_{2 n+1}(x)=\frac{\Gamma\left(\frac{5}{2}+m\right)}{(2 m+2) !} \sum_{r=0}^{m} \frac{(-1)^{r}\left(\begin{array}{c}
m \\
m-r
\end{array}\right)(2 m-r+2) !}{\Gamma\left(\frac{5}{2}+m-r\right)} x^{2 m-2 r+1} Y_{2 n+1}(x) .
$$

In virtue of the moment formula (30), the last formula turns into the form

$$
\begin{aligned}
Y_{2 m+1}(x) Y_{2 n+1}(x) \\
=\frac{\Gamma\left(\frac{5}{2}+m\right)}{(2+2 m) !} \sum_{r=0}^{m} \frac{(-1)^{r}\left(\begin{array}{c}
m \\
m-r
\end{array}\right)(2+2 m-r) !}{\Gamma\left(\frac{5}{2}+m-r\right)} \\
\quad \times \quad \sum_{\ell=0}^{2 m-2 r+1} \frac{(\ell+(1+n)(1+2 m-2 r))(2(m-r)) !}{2^{2 \ell}(1+n) \ell !(-\ell+2 m-2 r+1) !} Y_{2 m+2 n-2 r-2 \ell+2}(x) .
\end{aligned}
$$

Rearrangement of the terms in the right-hand side of (37), enables one to write

$$
\begin{aligned}
Y_{2 m+1}(x) Y_{2 n+1}(x) & \\
= & \frac{\left(\frac{3}{2}+m\right)}{(1+m)(1+n)} \sum_{p=0}^{2 m+1} \frac{1}{2^{2 p}} \\
& \quad \times \sum_{\ell=0}^{p} \frac{(-1)^{\ell}(1-3 \ell+n-2 \ell n+2 m(1+n)+p)(2-\ell+2 m) !}{(1-2 \ell+2 m)(3-2 \ell+2 m) \ell !(1-\ell+2 m-p) !(p-\ell) !} Y_{2 m+2 n-2 p+2}(x) .
\end{aligned}
$$


After some lengthy manipulation, the last linearization formula can be written in the following form:

$$
Y_{2 m+1}(x) Y_{2 n+1}(x)=\sum_{p=0}^{2 m+1} G_{p, m} Y_{2 m+2 n-2 p+2}(x)+\sum_{p=1}^{2 m+1} S_{p, m} Y_{2 m+2 n-2 p+2}(x),
$$

where

$$
G_{p, m}=\frac{(2 m) !(1+n+2 m(1+n)+p)}{2^{2 p}(1+n) p !(2 m-p+1) !}{ }_{3} F_{2}\left(\begin{array}{c}
-p,-\frac{3}{2}-m,-1-2 m+p \\
-2-2 m, \frac{1}{2}-m
\end{array} \mid 1\right)
$$

and

$$
\begin{aligned}
S_{p, m}= & \frac{(2 m) !(3+2 m)(3+2 n)(1+2 m-p) p}{2^{2 p+1}(1+n) p !(2 m-p+1) !\left(-1+m+2 m^{2}\right)} \\
& \times{ }_{3} F_{2}\left(\begin{array}{c}
1-p,-\frac{1}{2}-m,-2 m+p \\
-1-2 m, \frac{3}{2}-m
\end{array} \mid 1\right) .
\end{aligned}
$$

The ${ }_{3} F_{2}(1)$ that appears in the coefficient $G_{p, m}$ can be summed with the aid of the PfaffSaalschütz identity to give

$$
{ }_{3} F_{2}\left(\begin{array}{c}
-p,-\frac{3}{2}-m,-1-2 m+p \\
-2-2 m, \frac{1}{2}-m
\end{array} \mid 1\right)=\frac{(-1)^{p}(1+2 m)(p+1) !}{(1+2 m-2 p)(2 m-p+3)_{p}},
$$

while the ${ }_{3} F_{2}(1)$ that appears in the coefficient $S_{p, m}$ for all $p \geq 1$ can be summed with Zeilberger's and Petkovsek's algorithms (see [26]) to give

$$
\begin{aligned}
{ }_{3} F_{2} & \left(\begin{array}{c}
1-p,-\frac{1}{2}-m,-2 m+p \\
-1-2 m, \frac{3}{2}-m
\end{array} \mid 1\right) \\
& =\frac{\left(-1+2 m-2 p+(-1)^{p}(1-2 p(1+p)+2 m(3+2 p))\right) p !}{8 m(-1+2 m-2 p)(2 m-p)_{p-1}} .
\end{aligned}
$$

The reductions of the above two hypergeometric functions enable one to express explicitly the coefficients $G_{p, m}$ and $S_{p, m}$ in the following reduced forms:

$$
\begin{aligned}
& G_{p, m}=\frac{(-1)^{p}(1+p)(-2-2 m+p)(1+n+2 m(1+n)+p)}{2^{2 p+1}(1+m)(1+n)(-1-2 m+2 p)}, \\
& S_{p, m}=\frac{(3+2 m)(3+2 n)\left(-1-2 m+2 p+(-1)^{p}(1-2(-1+p) p+2 m(1+2 p))\right)}{2^{2 p+3}(1+m)(1+n)(-1-2 m+2 p)},
\end{aligned}
$$

and this leads to the following linearization formula:

$$
\begin{aligned}
Y_{2 m+1}(x) Y_{2 n+1}(x)= & \frac{1}{(m+1)(n+1)} \sum_{r=0}^{2 m+1} \frac{1}{2^{2 r+3}}\{(3+2 m)(3+2 n) \\
& \left.+(-1)^{r}\left(-1+2 m+2 n+4 m n-4(2+m+n) r+2 r^{2}\right)\right\} \\
& \times Y_{2 m+2 n-2 r+2}(x) .
\end{aligned}
$$




\title{
6 Conclusions
}

We have established some new formulas concerned with the sixth-kind Chebyshev polynomials. Connection formulas between the sixth-kind Chebyshev polynomials and some other orthogonal polynomials and their inversion formulas are derived with two different approaches. New moment formulas of the sixth-kind Chebyshev polynomials are also established. Based on the moment formulas, the linearization formulas of these polynomials are derived. Most of the formulas derived in this paper are new and useful to the best of our knowledge.

\begin{abstract}
Acknowledgements
The authors would like to thank the anonymous reviewers for carefully reading the article and also for their constructive
\end{abstract} and valuable comments, which have improved the paper in its present form.

Funding

There was no funding for this research.

Availability of data and materials

Not applicable.

Competing interests

The authors declare that they have no competing interests.

Authors' contributions

The two authors contributed equally to the work. Both authors read and approved the final manuscript.

\section{Publisher's Note}

Springer Nature remains neutral with regard to jurisdictional claims in published maps and institutional affiliations.

Received: 11 November 2020 Accepted: 18 January 2021 Published online: 28 January 2021

\section{References}

1. Gil, A., Segura, J., Temme, N.M.: Numerical Methods for Special Functions. SIAM, Philadelphia (2007)

2. Mason, J.C., Handscomb, D.C.: Chebyshev Polynomials. Chapman \& Hall/ CRC, Boca Raton (2003)

3. Ralston, A.: Numerical integration methods for the solution of ordinary differential equations. In: Ralston, A., Wilf, H.S. (eds.) Mathematical Methods for Digital Computers, vol. 1 (1960)

4. Gautschi, W.: On mean convergence of extended Lagrange interpolation. J. Comput. Appl. Math. 43(1-2), 19-35 (1992)

5. Masjed-Jamei, M.: Some new classes of orthogonal polynomials and special functions: a symmetric generalization of Sturm-Liouville problems and its consequences. Ph.D. thesis, Department of Mathematics, University of Kassel, Kassel, Germany (2006)

6. Abd-Elhameed, W.M., Youssri, Y.H.: Fifth-kind orthonormal Chebyshev polynomial solutions for fractional differential equations. Comput. Appl. Math. 37, 2897-2921 (2018)

7. Abd-Elhameed, W.M., Youssri, Y.H.: Sixth-kind Chebyshev spectral approach for solving fractional differential equations. Int. J. Nonlinear Sci. Numer. Simul. 20(2), 191-203 (2019)

8. Doha, E.H., Abd-Elhameed, W.M., Bassuony, M.A.: On the coefficients of differentiated expansions and derivatives of Chebyshev polynomials of the third and fourth kinds. Acta Math. Sci. 35(2), 326-338 (2015)

9. Doha, E.H., Abd-Elhameed, W.M.: On the coefficients of integrated expansions and integrals of Chebyshev polynomials of third and fourth kinds. Bull. Malays. Math. Sci. Soc. 37(2), 383-398 (2014)

10. Lee, K.: Chebyshev collocation method for the constant mobility Cahn-Hilliard equation in a square domain. Appl. Math. Comput. 370, Aticle ID 124931 (2020)

11. Babaei, A., Jafari, H., Banihashemi, S.: Numerical solution of variable order fractional nonlinear quadratic integro-differential equations based on the sixth-kind Chebyshev collocation method. J. Comput. Appl. Math. 377 , Aticle ID 112908 (2020)

12. Ahmed, H.M.: Computing expansions coefficients for Laguerre polynomials. Integral Transforms Spec. Funct. (2020). https://doi.org/10.1080/10652469.2020.1815727

13. Abd-Elhameed, W.M., Ali, A.: New specific and general linearization formulas of some classes of Jacobi polynomials. Mathematics 9(1), Article ID 74 (2021)

14. Abd-Elhameed, W.M.: New formulae for the linearization coefficients of some nonsymmetric Jacobi polynomials. Adv. Differ. Equ. 2015, 168 (2015)

15. Chaggara, H., Koepf, W.: On linearization coefficients of Jacobi polynomials. Appl. Math. Lett. 23(5), 609-614 (2010)

16. Kim, T., Kim, D.S., Jang, L.-C., Dolgy, D.V.: Representation by Chebyshev polynomials for sums of finite products of Chebyshev polynomials. Symmetry 10(12), Article ID 742 (2018)

17. Kim, T., Kim, D.S., Jang, L.-C., Dolgy, D.V.: Representing by several orthogonal polynomials for sums of finite products of Chebyshev polynomials of the first kind and Lucas polynomials. Adv. Differ. Equ. 2019, 162 (2019)

18. Kim, T., Kim, D.S., Dolgy, D.V., Park, J.-W.: Sums of finite products of Legendre and Laguerre polynomials. Adv. Differ. Equ. 2018, 277 (2018) 
19. Kim, T., Kim, D.S., Lee, H., Kwon, J.: Studies in sums of finite products of the second, third, and fourth kind Chebyshev polynomials. Mathematics 8(2), Article ID 210 (2020)

20. Abd-Elhameed, W.M., Youssri, Y.H., El-Sissi, N., Sadek, M.: New hypergeometric connection formulae between Fibonacci and Chebyshev polynomials. Ramanujan J. 42, 347-361 (2017)

21. Dolgy, D.V., Kim, D.S., Kim, T., Kwon, J.: Connection problem for sums of finite products of Chebyshev polynomials of the third and fourth kinds. Symmetry 10(11), Article ID 6170 (2018)

22. Tcheutia, D.D.: On connection, linearization and duplication coefficients of classical orthogonal polynomials. Ph.D. thesis, University of Kassel, Kassel, Germany (2014)

23. Abd-Elhameed, W.M.: New product and linearization formulae of Jacobi polynomials of certain parameters. Integral Transforms Spec. Funct. 26(8), 586-599 (2015)

24. Abd-Elhameed, W.M., Doha, E.H., Ahmed, H.M.: Linearization formulae for certainJacobi polynomials. Ramanujan J. 39(1), 155-168 (2016)

25. Doha, E.H., Abd-Elhameed, W.M.: Efficient spectral-Galerkin algorithms for direct solution of second-order equations using ultraspherical polynomials. SIAM J. Sci. Comput. 24(2), 548-571 (2002)

26. Koepf, W.: Hypergeometric Summation. An Algorithmic Approach to Summation and Special Function Identities. Springer, London (2014)

27. Olver, F.W.J., Lozier, D.W., Boisvert, R.F., Clark, C.W.: NIST Handbook of Mathematical Functions. Cambridge University Press, Cambridge (2010). DLMF, Digital Library of Mathematical Functions

\section{Submit your manuscript to a SpringerOpen ${ }^{\circ}$ journal and benefit from:}

- Convenient online submission

- Rigorous peer review

- Open access: articles freely available online

- High visibility within the field

- Retaining the copyright to your article

Submit your next manuscript at $\mathbf{s p r i n g e r o p e n . c o m ~}$ 